\title{
Relação entre indicadores de gestão pública e de desenvolvimento dos municípios brasileiros
}

\section{Relationship between public administration indicators and the development of Brazilian municipalities \\ Relación entre los indicadores de la administración pública y el desarrollo de las ciudades brasileñas}

Resumo: 0 objetivo principal desta pesquisa foi avaliar a relação entre os indicadores de gestão pública (IFGF - Índice Firjan de Gestão Fiscal) e os de desenvolvimento dos municípios brasileiros (IFDM - Índice Firjan de Desenvolvimento Municipal). Os dados foram de mensuração anual, secundários, dispostos em um painel referente aos anos de 2006 a 2011. Observaram-se, nos municípios brasileiros, nível de desenvolvimento médio moderado e indicadores de gestão pública municipal em dificuldade crítica, excluindo-se 0 custo da dívida e gastos com pessoal, variáveis que foram analisadas pela Lei de Responsabilidade Fiscal ao longo do tempo. Verificou-se que os municípios apresentam oscilações temporais nos investimentos públicos municipais, em períodos cíclicos cujas trajetórias coincidem com períodos antes das eleições. Os resultados das regressões de dados em painel evidenciaram que o desenvolvimento municipal foi explicado em 33,75\% pelo conjunto dos indicadores de gestão pública municipal com PIB Per capita municipal.

Palavras-chave: Gestão pública municipal, desenvolvimento, lei de responsabilidade fiscal, indicadores, municípios brasileiros

Geraldo Alemandro Leite Filho - geraldo.alemandro@unimontes.br

Professor da Universidade Estadual de Montes Claros, Departamento de Ciências Contábeis, Montes Claros, MG, Brasil.

Tânia Marta Maia Fialho - tania.unimontes@gmail.com

Professora da Universidade Estadual de Montes Claros, Departamento de Economia, Montes Claros, MG, Brasil.

Artigo submetido no dia 24-06-2015 e aprovado em 17-11-2015.

DOI: http://dx.doi.org/10.12660/cgpc.v20n67.52080 


\section{Abstract}

The objective of this research was to evaluate the relationship between governance indicators (IFGF - Fiscal Management FIRJAN Index) and the development indicators of Brazilian municipalities (IFDM - Municipal Development FIRJAN Index). Annual measurement data were used, arranged in a panel, and considered the years 2006 to 2011. Brazilian cities were seen to have average levels of moderate development and municipal management indicators in difficulty criticism, excluding debt cost and personnel expenses, the two variables that were most closely monitored by the Fiscal Responsibility Law over time. It was found that there are seasonal and cyclical changes in municipal public investments, whose trajectories coincide with periods before elections. The results of the panel data regressions showed that municipal development was explained in $33.75 \%$ of the cases by all the indicators of municipal management with municipal GDP.

Keywords: Municipal Public Management, Development, Fiscal Responsibility Law, Indicators, Brazilian Municipalities

\section{Resumen}

El principal objetivo de esta,investigación fue evaluar la relación entre los indicadores de gobernabilidad (IFGF - Gestión Fiscal Indice FIRJAN), los indicadores de desarrollo de los municipios brasileños (IFDM - Indice de Desarrollo Municipal FIRJAN). Los datos fueron la medición anual, secundarios, dispuestos en un panel referente a los años 2006 a 2011. Se observa en las ciudades brasileñas, nivel medio de desarrollo e indicadores de gestión municipal en crisis la crítica moderada, excluyendo el costo deuda y gastos de personal, variables que fueron más seguidas por la Ley de Responsabilidad Fiscal en el tiempo. Se encontró que los municipios tienen los cambios estacionales en las inversiones públicas municipales en períodos cíclicos cuyas trayectorias coinciden con períodos anteriores a las elecciones. Los resultados de las regresiones de datos de panel mostraron que el desarrollo municipal se explicó en el 33,75 \% en todos los indicadores de la gestión municipal con un PIB per cápita municipal.

Palabras clave: la gestión pública de la ciudad, desarrollo, Ley de Responsabilidad Fiscal, Indicadores, ciudades brasilenas.

\section{INTRODUÇÃO}

Durante anos, os municípios brasileiros enfrentaram problemas tais como inflação elevada, recessão econômica, carência de recursos para investimentos e, no âmbito interno, ausência de instrumentos eficazes de planejamento e controle dos gastos públicos municipais, fatores estes que impactaram negativamente na gestão e no desempenho econômico e social dos municípios. Além disso, a história da administração pública no Brasil, principalmente em nível municipal, está repleta de exemplos de má gestão de recursos, tendo como agravantes a falta de responsabilidade e compromisso social de alguns gestores públicos, o que colaborou para elevados índices de corrupção e endividamento, devido, principalmente, à ausência de uma regulação mais severa de controle na gestão pública municipal brasi- leira (Meirelles, 2002; Oliveira, Peter \& Meneses, 2010; Sousa et al., 2011; Oliveira \& Silva, 2012; Klering, Kruel \& Stranz, 2012; Sousa et al., 2013; Duarte \& Lima 2013; Denardi et al., 2014).

Nesse contexto, diversas experiências exitosas de outros países no planejamento e controle da gestão pública serviram de referência para o Brasil, propiciando o debate e a construção de mecanismos sobre o controle nos gastos públicos e a responsabilização dos gestores acerca dos recursos públicos sob a sua guarda (Sousa et. al., 2011). O controle da inflação, estabilidade monetária e maior transparência nos resultados fiscais governamentais obtidos a partir do Plano Real contribuíram também para fortalecer o debate sobre a necessidade de ajustes nas contas públicas e para a criação de ferramentas com o propósito de planejar 
e controlar os gastos públicos e obter uma gestão pública eficiente (Matias, Ortolani \& Campello, 2002).

Em 4 de maio de 2000, foi sancionada no Brasil a Lei Complementar 101, denominada Lei de Responsabilidade Fiscal (LRF), que impôs rigor ao processo de planejamento e execução orçamentária, disciplinando a gestão dos recursos públicos e exigindo maior responsabilidade e transparência dos gestores públicos. Tal lei limitou os gastos e endividamentos dos entes, buscou equilíbrio entre receitas e despesas públicas e fomento de investimentos prioritários ao desenvolvimento e melhora dos indicadores sociais (Oliveira et al., 2010). Segundo Sousa et al. (2011), quando se trata da gestão pública municipal, a perspectiva da LRF torna-se ainda mais relevante, pois, diante da municipalização, da escassez de recursos para investimentos e da crescente demanda por serviços sociais, como saúde, educação, segurança e lazer. A referida lei teve o objetivo principal de intensificar 0 controle e a responsabilização dos gestores municipais mediante as contas públicas, objetivando eficiência na alocação de recursos e otimização de custos da máquina pública e, consequentemente, o desenvolvimento, direcionando os recursos públicos em investimentos que melhorem a qualidade de vida das pessoas.

Entende-se que a melhora dos indicadores de desenvolvimento está relacionada à maior eficiência da gestão pública. A eficiência, considerada um princípio constitucional, pode ser definida, segundo Meirelles (2002), como o que se impõe a todo agente público: realizar suas atribuições com presteza, perfeição e rendimento funcional, exigindo resultados positivos para o serviço público e satisfatório atendimento das necessidades da comunidade e de seus membros.

A busca pela eficiência na gestão pública também pauta-se por efetivo controle dos gastos públicos e transparência na aplicação dos recursos, temas contidos na LRF. Nessa perspectiva, desde a criação da LRF, diversos estudos foram feitos para verificar se o cumprimento de suas prerrogativas, pelos entes federativos brasileiros, impactou os indicadores de desenvolvimento, principalmente no âmbito municipal, destacando-se Matias et al. (2002); Resende, Slomski, e Corrar (2005); Oliveira et al., (2010); Sousa et al., (2011); Oliveira e Silva (2012); Klering, Kruel, e Stranz (2012); Sousa et al., (2013); Duarte e Lima (2013); Denardi et al., (2014), Leite Filho e Fialho (2014).

Diante do contexto apresentado, o objetivo principal desta pesquisa foi verificar a relação entre indicadores de gestão pública municipal, com base na LRF e nos indicadores de desenvolvimento municipal dos municípios brasileiros. Como objetivos secundários, este estudo buscou: a) analisar e discutir descritivamente a evolução dos indicadores de gestão municipal e de desenvolvimento municipal, bem como avaliar efeitos espaciais e temporais; b) verificar a correlação entre os indicadores; c) examinar a relação entre os indicadores de gestão pública municipal e indicadores de desenvolvimento municipal por meio de regressão de dados em painel.

Acredita-se ser importante relacionar indicadores de gestão pública municipal, com base na LRF, com os indicadores de desenvolvimento municipal, para testar a as- 
sertiva de que aspectos da gestão pública municipal poderiam estar associados aos indicadores de desenvolvimento municipal, numa perspectiva temporal mais ampla. Tal investigação contribui para complementar a discussão e o entendimento de aspectos das finanças públicas municipais, responsabilidade fiscal e desenvolvimento municipal, temas discutidos nos estudos anteriormente realizados.

A principal contribuição deste trabalho é verificar a intensidade da relação entre os indicadores de gestão pública municipal e os indicadores de desenvolvimento municipal, numa perspectiva de abrangência nacional e temporal de médio prazo, com dados de todos os municípios brasileiros em uma série histórica de seis anos consecutivos.

Este estudo está estruturado em cinco seções, incluindo esta introdução. A segunda seção discorre sobre os conceitos teóricos de responsabilidade fiscal e desenvolvimento municipal. Na terceira, é apresentada a metodologia utilizada no desenvolvimento desta pesquisa. A quarta apresenta os resultados encontrados e a quinta consiste na conclusão. Em seguida, têm-se as referências.

\section{Referencial teórico}

A ação do Estado-nação se efetiva por meio da gestão pública, objetivando viabilizar e garantir direitos, ofertar serviços e distribuir recursos. Nesse processo, o principal desafio dos governos e da administração pública é promover o desenvolvimento econômico e social sustentável, num ambiente de mudanças de paradigmas, que estão impactando de maneira profunda a socieda- de, em especial nas áreas socioeconômica, ambiental, cultural e tecnológica. Além da preocupação permanente em elevar o nível do desempenho na gestão pública, deve-se dedicar especial atenção às questões que envolvem ética, moral e transparência na administração pública (Pereira, 2012).

Dessa forma, buscando maior responsabilidade e transparência na gestão fiscal, surgiu a Lei de Responsabilidade Fiscal - LRF - (Lei Complementar n. 101/2000) em um contexto no qual era necessário impor limites e metas numéricas aos gestores da política fiscal, nos vários níveis de governo, a fim de se gerar menores deficits fiscais e menor acúmulo de dívida, entre outros resultados sinalizadores de uma atitude fiscal responsável, o que levou à criação, no Brasil, do conceito de Responsabilidade Fiscal na Gestão pública (Firovante, Pinheiro \& Vieira, 2006).

A LRF está apoiada em quatro eixos: planejamento, no qual são estabelecidas as metas a serem atingidas durante a gestão; transparência, que consiste na ampla divulgação das contas da administração pública; controle, que se refere ao monitoramento realizado tanto pelos órgãos competentes quanto pela sociedade, e responsabilização, que são as sanções previstas pela lei, em decorrência da má utilização dos recursos públicos (Sousa et al., 2013).

Segundo Viana et al. (2011), é preciso evidenciar a qualidade da gestão pública, principalmente dos gastos públicos, desde que os limites previstos nas legislações não sejam ultrapassados, destacando recursos públicos para a garantia dos direitos sociais preconizados pela Constituição brasileira. Assim, com o intuito de observar o com- 
portamento dos municípios, em relação ao controle de gastos, investimentos e cumprimento dos direitos sociais estabelecidos na Constituição, foram criados indicadores para medir o desempenho dos municípios no que se refere à gestão pública municipal e ao desenvolvimento econômico e social (Sousa et al., 2013).

Jannuzzi (2002, p. 55) classifica "indicador como uma medida em geral quantitativa, dotada de significado social substantivo, usado para operacionalizar um conceito social abstrato, que informa algo sobre um aspecto da realidade social ou sobre mudanças que estão ocorrendo na mesma." Segundo o autor, são importantes na gestão pública, pois se prestam a subsidiar as atividades de planejamento público e a formulação de políticas sociais, bem como avaliar bem-estar e os determinantes dos diferentes fenômenos sociais.

Para Kayano e Caldas (2002), indicadores são instrumentos importantes para fiscalização, controle, gestão, verificação e medição de eficiência e eficácia na administração pública, por permitirem comparar situações entre localidades (espaços territoriais) ou entre períodos diferentes de um mesmo município.

Jannuzzi (2001) chama a atenção para a utilização dos indicadores para avaliação econômica e social da gestão pública. Para o autor, o conhecimento do significado, dos limites e das potencialidades dos indicadores sociais pode ser de grande utilidade para os diversos agentes e instituições envolvidos na definição das prioridades sociais e na alocação de recursos do orçamento público. $O$ autor observa que os indicadores podem orientar a análise, a formulação e a implementação de políticas sociais e contribuir para o apontamento da magnitude das carências a atender nas diversas áreas de intervenção por parte da gestão pública.

Nahas et al. (2006) destacam que o Brasil tem se avançado na produção de indicadores municipais, o que demonstra uma preocupação da gestão estadual e municipal no uso de indicadores para gestão e planejamento urbano e regional. Os autores destacam a existência de grande variabilidade de metodologias, o que possibilita o desenvolvimento de estudos e sistemas adequados à diversidade cultural que reflitam as condições de vida existentes nas diferentes regiões brasileiras.

Segundo Guimarães (2008), para a avaliação da gestão, indicadores compostos são importantes porque permitem fazer comparações globais da situação do município e do desempenho da gestão. A disponibilidade de indicadores para uso no diagnóstico da realidade social empírica, formulação de políticas, monitoramento das condições de vida da população, análise da mudança social está, pois, condicionada à oferta e às características das estatísticas públicas.

Para Guimarães e Jannuzzi (2004), o avanço dos processos de descentralização, a maior pressão social oriunda de sucessivas crises econômicas e o consequente acúmulo de demandas locais vêm fazendo com que a geração e a disseminação periódica de estatísticas, indicadores e índices municipais passem a assumir um caráter ainda mais relevante no processo de planejamento, sobretudo para subsidiar o processo de implantação, monitoramento e avaliação das políticas públicas. 
Segundo Silva (1995), para que os municípios assumam e atendam as diversas demandas sociais e econômicas, há necessidade de se desenvolver instrumentos e indicadores de gestão pública, com o objetivo de avaliar o desempenho da gestão e medir os níveis de desenvolvimento econômico e social. Por esse motivo, destacam-se os indicadores da Federação das Indústrias do Estado do Rio de Janeiro (Firjan).

Do ponto de vista dos indicadores de gestão pública municipal, o Índice Firjan de Gestão Fiscal (IFGF), desenvolvido pela Gerência de Estudos Econômicos da Firjan, tem como objetivo estimular a cultura da responsabilidade administrativa, por meio da geração e divulgação de indicadores que possibilitem aperfeiçoar as decisões dos gestores municipais quanto à alocação dos recursos públicos, bem como gerar maior controle social da gestão fiscal dos municípios (Firjan, 2014).

O índice IFGF é construído a partir dos resultados fiscais das próprias prefeituras - informações de declaração obrigatória, dados de receitas, despesas, ativos e passivos - e disponibilizadas anualmente pela Secretaria do Tesouro Nacional (STN) e da base de dados do Finanças do Brasil (Finbra). Coleta ainda informações das contas nacionais e do IBGE. A pontuação do índice varia entre os extremos 0 (pior situação) e 1 (melhor situação). Classifica as gestões municipais em quatro categorias de qualidade: A (Gestão de Excelência, com resultados superiores a 0,8), B (Boa Gestão, entre 0,6 e 0,8), C (Gestão em dificuldade, entre 0,4 e 0,6 ) e D (Gestão crítica, abaixo de 0,4$)$.
Klering, Kruel, e Stranz (2012) observam que o IFGF da Firjan avalia essencialmente indicadores fiscais e inclui na avaliação o indicador Receita Própria, avaliando a capacidade de geração de receita própria dos municípios, variável que está ligada diretamente à capacidade de gestão e ao nível de desenvolvimento econômico e social dos municípios.

Com relação aos indicadores de desenvolvimento econômico e social, o Índice Firjan de Desenvolvimento Municipal (IFDM), administrado pela Firjan, monitora anualmente o desenvolvimento dos municípios do Brasil nas áreas emprego e renda, educação e saúde, com base nas informações disponibilizadas pelos Ministérios do Trabalho, da Educação e da Saúde. A variação do IFDM é de 0 a 1 , considerando-se que quanto mais próximo de 1 estiver o município, maior é o seu desenvolvimento. Classifica o nível de desenvolvimento municipal em Muito alto $(0,9001 \mathrm{a}$ $1,0)$, Alto $(0,8001$ a 0,9$)$, Moderado $(0,6001$ a $0,8)$, Regular $(0,4001$ a 0,6$)$ e Baixo $(0$ a 0,4$)$ (Firjan, 2014; Souza et al., 2013).

O Índice Firjan de Desenvolvimento Municipal (IFDM) é referência para o acompanhamento do desenvolvimento socioeconômico municipal ao longo de séries históricas anuais. O IFDM apresenta semelhanças com o IDH-M, pois acompanham as três áreas de seu interesse: renda, educação e saúde, utilizando-se exclusivamente de estatísticas oficiais (Ervilha, Alves \& Gomes, 2013).

Oliveira e Silva (2012) entendem que o desenvolvimento econômico municipal é caracterizado pelo crescimento da renda, acompanhado de melhorias no nível de qualidade vida da população. As variáveis renda per 
capita e Índice de Desenvolvimento Humano (IDH) apresentam grande aceitação na literatura como importantes indicadores para avaliação do nível de desenvolvimento de um município, localidade, região ou país. Asseveram que, embora a renda seja um relevante indicador, apontam-se outros aspectos ligados à qualidade de vida, tais como saúde e educação, como variáveis chave na medição do desenvolvimento municipal.

Apesar de os estudos realizados no Brasil associarem a questão do desenvolvimento às variáveis de renda, Costa (2011) entende que ela seja subdimensionada, pois a dimensão renda corresponde a apenas um dos parâmetros referentes à situação dos indivíduos na sociedade. $\mathrm{O} I \mathrm{IH}$, por outro lado, como importante instrumento de medição do desenvolvimento, tem a vantagem de abordar as variáveis educação e saúde, mas apresenta uma limitação temporal para avaliar os municípios em séries temporais por ser um indicador deceanual (Oliveira \& Silva, 2012).

Segundo Mendes e Rezende (2008), a utilização e a análise dos indicadores municipais podem permitir, aos municípios, melhor avaliação dos planos a serem executados para proporcionar um crescimento econômico nas regiões pesquisadas, bem como comparações temporais com outros municípios.

Siedenberg (2003) defende o uso de indicadores para análise e avaliação dos pontos fortes e fracos, assim como do sucesso ou fracasso de uma estratégia de gestão ou de desenvolvimento de uma cidade ou região. Corroborando com Jannuzzi (2001), a utilização de indicadores em séries temporais históricas regulares é necessária para permitir análises e comparações municipais inter ou intra-regionais e a evolução do município em termos temporais. Nesse contexto, por se tratar de indicadores com medições baseadas em estatísticas oficiais e por terem uniformidade e regularidade ao longo do tempo, verifica-se que o IFGF e o IFDM podem ser indicadores eficientes de avaliação dos aspectos da gestão municipal bem como do desenvolvimento.

\section{Metodologia da pesquisa}

Caracteriza-se esta pesquisa quanto à abordagem do problema de natureza de avaliação quantitativa de dados e aos objetivos. Trata-se de um estudo descritivo, uma vez que foi realizado um levantamento documental com coleta, tratamento, análise e interpretação de dados secundários.

As variáveis de interesse da pesquisa foram coletadas da base de dados da Firjan. Como variável para medir a qualidade da gestão pública municipal, foi utilizado o Índice Firjan de Gestão Fiscal (IFGF) subdividido nas seguintes dimensões: a) IFGF Receita Própria; b) IFGF Gastos com Pessoal; c) IFGF Investimentos; d) IFGF Liquidez; e) IFGF Custo da Dívida. A variável para a medida do desenvolvimento municipal foi o Índice Firjan de Desenvolvimento Municipal (IFDM), sendo função dos subíndices IFDM Emprego e Renda, IFDM Educação e IFDM Saúde. As variáveis IFGF e IFDM foram coletadas de forma fidedigna, sem qualquer alteração ou correção, apresentando-se como variáveis quantitativas contínuas. A variável PIB Per capita Municipal foi coletada da base de dados do IBGE para todos os municípios brasileiros e foi corrigida em valores de 2011. O 


\section{quadro 1 apresenta a definição das variáveis utilizadas na pesquisa.}

\begin{tabular}{|c|c|c|}
\hline Tipo & Variável & Descrição \\
\hline Dependente & $\begin{array}{l}\text { IFDM - índice Firjan de desenvol- } \\
\text { vimento municipal }\end{array}$ & $\begin{array}{l}\text { Avalia o desenvolvimento socioeconômico de todos os municípios brasileiros, } \\
\text { sendo uma função de três subíndices: Emprego e renda, Educação e Saúde. } \\
\text { Calculado com base em estatísticas públicas oficiais, disponibilizadas pelos mi- } \\
\text { nistérios do Trabalho, Educação e Saúde. Mensurado anualmente, varia de } 0 \\
\text { (pior) até 1,0 (melhor). }\end{array}$ \\
\hline $\begin{array}{l}\text { Regressor - Vari- } \\
\text { ável Independente }\end{array}$ & $\begin{array}{l}\text { IFGF - índice Firjan de Gestão } \\
\text { Fiscal - Receita Própria }\end{array}$ & $\begin{array}{l}\text { Avalia o grau de autonomia das receitas dos municípios, relacionando Receita } \\
\text { Própria }(\mathrm{RP}) \text { / Receita Corrente Líquida (RCL). Para municípios que geraram até } \\
50 \% \text { da relação RP / RCL, recebem nota máxima (1), os demais recebem nota } \\
\text { proporcional entre } 1 \text { e } 0 \text {. Para os que não geraram nada, nota zero. Mensurado } \\
\text { anualmente, varia de } 0 \text { (pior) até } 1,0 \text { (melhor). }\end{array}$ \\
\hline $\begin{array}{l}\text { Regressor - Vari- } \\
\text { ável Independente }\end{array}$ & $\begin{array}{l}\text { IFGF - índice Firjan de Gestão } \\
\text { Fiscal - Gastos com pessoal }\end{array}$ & $\begin{array}{l}\text { A LRF limitou gastos com pessoal em } 60 \% \text { da RCL. Esse indicador avalia o com- } \\
\text { prometimento das } \mathrm{RCL} \text { com os gastos de pessoal (GP). Municípios que supe- } \\
\text { ram } 60 \% \text { da relação GP/RCL recebem nota zero, com relação menor que } 30 \% \text {, } \\
\text { recebem nota 1, os demais obtém notas proporcionais entre } 1 \text { e } 0 \text {. Quanto mais } \\
\text { próximo de um melhor, menor o comprometimento do orçamento municipal com } \\
\text { GP. Mensurado anualmente, varia de } 0 \text { (pior) até } 1,0 \text { (melhor). }\end{array}$ \\
\hline $\begin{array}{l}\text { Regressor - Vari- } \\
\text { ável Independente }\end{array}$ & $\begin{array}{l}\text { IFGF - índice Firjan de Gestão } \\
\text { Fiscal - Investimentos }\end{array}$ & $\begin{array}{l}\text { Visa medir a capacidade dos investimentos públicos no orçamento municipal. } \\
\text { Relaciona o Investimento (I) com Receita Corrente Líquida (RCL). Para municí- } \\
\text { pios com essa relação acima de } 20 \% \text {, nota } 1 \text {, para os demais proporcionalmente } \\
\text { variando de } 1 \text { a } 0 \text {. Mensurado anualmente, varia de } 0 \text { (pior) até 1,0 (melhor). }\end{array}$ \\
\hline $\begin{array}{l}\text { Regressor - Vari- } \\
\text { ável Independente }\end{array}$ & $\begin{array}{l}\text { IFGF - índice Firjan de Gestão } \\
\text { Fiscal - Liquidez }\end{array}$ & $\begin{array}{l}\text { A LRF estabeleceu que, no último ano de mandato, a gestão municipal tenha } \\
\text { recursos financeiros suficientes para fazer frente ao montante de restos a pagar. } \\
\text { Assim, o indicador mede a relação dívidas e ativos financeiros ( } 1 \text { - Restos a } \\
\text { Pagar/Ativos Financeiros). Varia de } 0 \text { a 1, quanto mais próximo de } 1 \text {, menos o } \\
\text { município está postergando pagamentos sem cobertura, melhor sua situação de } \\
\text { liquidez. Mensurado anualmente, varia de } 0 \text { (pior) até 1,0 (melhor). }\end{array}$ \\
\hline $\begin{array}{l}\text { Regressor - Vari- } \\
\text { ável Independente }\end{array}$ & $\begin{array}{l}\text { IFGF - índice Firjan de Gestão } \\
\text { Fiscal - Custo da Dívida }\end{array}$ & $\begin{array}{l}\text { Avalia o peso dos encargos da dívida em relação às receitas líquidas. Conforme } \\
\text { resolução } 43 \text { do Senado Federal, a relação Dívidas / Receitas Líquidas tem o } \\
\text { limite de } 13 \% \text {. Para municípios com relação maior que } 13 \% \text {, recebem nota } 0 \text {, } \\
\text { para os demais, notas escalonadas variando de } 0 \text { a 1. Quanto menor a relação, } \\
\text { maior o índice. Mensurado anualmente, varia de } 0 \text { (pior) até 1,0 (melhor). }\end{array}$ \\
\hline $\begin{array}{l}\text { Regressor - Variá- } \\
\text { vel de controle }\end{array}$ & PIB Per capita Municipal & $\begin{array}{l}\text { Fornece o valor adicionado da agropecuária, indústria e serviços, a preços cor- } \\
\text { rentes, por meio de um processo descendente de repartição, pelos municípios, } \\
\text { do valor adicionado das } 15 \text { atividades econômicas das Contas Regionais obtido } \\
\text { para cada Unidade da Federação. Mensurado anualmente, foi apresentado lo- } \\
\text { garitimizado pelo seu valor corrigido a valores de } 2011 \text {. }\end{array}$ \\
\hline
\end{tabular}

\section{Quadro 1. Descrição das variáveis usadas na pesquisa Fonte: elaborado pelos autores}


O universo da pesquisa foi definido com todos os 5.570 municípios brasileiros. Para a pesquisa, foram selecionados aqueles que tiveram os indicadores IFGF e IFDM calculados anualmente pela Firjan, anos de referência 2006, 2007, 2008, 2009, 2010 e 2011. Observou-se que muitos municípios brasileiros não tiveram os indicadores IFGF calculados, em razão da falta de informações ou de dados incorretos. Assim, foram obtidas 31421 observações válidas, distribuídas em uma amostra de 5.555 municípios com informações completas de todos os indicadores de interesse da pesquisa, correspondendo a $99,73 \%$ do universo do estudo, classificados em um painel de dados contendo seis séries anuais empilhadas e desbalanceadas. Os municípios excluídos foram aqueles que não enviaram as informações ou que os dados estavam incompletos, não sendo possível o cálculo dos indicadores de interesse da pesquisa pela Firjan. Foi pos- sível, portanto, analisar em um só modelo o comportamento do conjunto das variáveis, IFGF e IFDM, para todas as observações, no período pesquisado, especificado pelas dimensões municipais e anuais.

O método para análise dos dados foi a regressão de dados em painel estático. Dados em painel ou dados longitudinais são caracterizados por contarem com observações em duas dimensões que, em geral, são o tempo e o espaço (Gujarati, 2006). Os modelos de regressão de dados em painel estático foram processados nas três abordagens: efeito pooling, efeitos fixos (EF) e efeitos aleatórios (EA) (Wooldridge, 2002, \& Gujarati, 2006). O modelo geral usado para verificar os efeitos dos indicadores de qualidade da gestão pública municipal nos municípios brasileiros é relatado a seguir, pela seguinte equação:

\footnotetext{
IFDM $_{\text {it }}=\alpha i+\beta 0^{*}$ IFGF Receita Própriait $+\beta 1^{*}$ IFGF Gastos com Pessoalit + ß2*IFGF Investimentosit + ß3*IFGF Liquidezit + $34^{*} I F G F$ Custo da Dívidait $+\beta 5^{*}$ PIB Municipalit $+\mu \mathrm{i}+$ nit
}

com $\mathrm{i}=1, \ldots, \mathrm{N}$ e $\mathrm{t}=1, \ldots, \mathrm{T}$

Com relação aos critérios de confiabilidade, os dados para cálculo dos indicadores IFGF e IFDM foram coletados de forma autêntica, sem alterações, da base de dados da Firjan. Tais indicadores foram usados em estudos anteriores, destacando-se Oliveira e Silva (2012); Klering, Kruel, e Stranz (2012); Sousa et al., (2013); Duarte e Lima (2013). Entende-se serem fidedignos. Os dados foram organizados, tratados e analisados por meio do software Stata 11.0.
Inicialmente, processou-se uma análise descritiva das variáveis de interesse da pesquisa com os objetivos de verificar não somente a medida de tendência central e a dispersão, como também se as variações entre os municípios (between) foram maiores ou menores que as variações ao longo do tempo (within), ou seja, se o efeito espacial (interação espacial entre os municípios) fora mais evidente do que o efeito temporal nos indicadores dos municípios. A tabela 1 apresenta os resultados

\section{Resultados}


Tabela 1. Resultados da análise descritiva das variáveis

\begin{tabular}{|c|c|c|c|c|c|c|}
\hline \multirow{4}{*}{ IFDM } & \multicolumn{2}{|r|}{ Média } & Desv. Pad & \multirow{4}{*}{$16,78 \%$} & \multirow{4}{*}{$\begin{array}{l}\text { Min } \\
0,2553417\end{array}$} & \multirow{4}{*}{$\begin{array}{l}\text { Máx } \\
0,9524284\end{array}$} \\
\hline & Overall & 0,6283 & 0,1054049 & & & \\
\hline & Between & & 0,981316 & & & \\
\hline & Within & & 0,0410884 & & & \\
\hline Receita & Overall & 0,234452 & 0,1905306 & $81,27 \%$ & 0,0021108 & 1 \\
\hline Própria & Between & & 0,1624981 & & & \\
\hline & Within & & 0,0981763 & & & \\
\hline Gastos & Overall & 0,6342815 & 0,1903263 & $30,01 \%$ & 0 & 1 \\
\hline com pessoal & Between & & 0,1355701 & & & \\
\hline & Within & & 0,1351785 & & & \\
\hline Investi- & Overall & 0,556272 & 0,281599 & $50,62 \%$ & 0 & 1 \\
\hline mentos & Between & & 0,1791462 & & & \\
\hline & Within & & 0,2185334 & & & \\
\hline Liquidez & Overall & 0,5675127 & 0,3724785 & $65,63 \%$ & 0 & 1 \\
\hline & Between & & 0,2647619 & & & \\
\hline & Within & & 0,2634557 & & & \\
\hline Custo da & Overall & 0,7995585 & 0,1738581 & $21,74 \%$ & 0 & 1 \\
\hline Dívida & Between & & 0,1271452 & & & \\
\hline & Within & & 0,1190457 & & & \\
\hline PIB & Overall & 11,72971 & 1,388055 & $11,83 \%$ & 8,678121 & 20,04601 \\
\hline Per capita & Between & & 1,339134 & & & \\
\hline & Within & & 0,3408259 & & & \\
\hline
\end{tabular}

Fonte: elaborado pelos autores

Observou-se uma média de 0,6283 do IFDM com variabilidade de $16,78 \%$, classificado como desenvolvimento médio moderado. Com relação aos indicadores de gestão pública municipal, as maiores médias foram observadas nos indicadores Custo da dívida (média de 0,7996 e variabilidade de $21,73 \%$ ) e Gastos com pessoal (média de 0,6342 com variabilidade de $30,00 \%$ ), classificados como indicadores médios de Boa gestão. Os indicadores de Liquidez (média de 0,5676 e variabilidade de $65,62 \%$ ) e Investimentos (média de 0,5563 e variabilidade de $50,62 \%$ ) foram classificados em Gestão em dificuldade. Já o indicador Receita própria (média de 0,2344 e variabilidade de $81,27 \%$ ) apresentou menor média e maior desvio padrão, denotando Gestão crítica. De uma maneira geral, a perspectiva temporal dos municípios brasileiros de 2006 a 2011 é de desenvolvimento moderado, e os indicadores de qualidade de gestão são considerados de gestão em dificuldade à crítica, excluindo-se o custo da dívida e gastos com pessoal, variáveis que são mais observadas pela LRF.

Com relação aos efeitos espacial e temporal, as variáveis que demonstraram maior variação entre os municípios (between) foram o 
IFDM, IFGF - Receita própria, IFGF - Liquidez, IFGF - Custo da dívida e o PIB Per capita, o que reflete a heterogeneidade entre os municípios brasileiros. Para todas as variáveis da pesquisa, excluindo-se o IFGF - Liquidez, verificou-se que houve um efeito espacial (interação espacial entre os municípios) mais significativo do que a influência temporal entre as variáveis da pesquisa, ou seja, os municípios se influenciam. Observou-se que a variável IFGF Investimentos foi a única que apresentou uma variação temporal maior ao longo do tempo (within) em comparação à interação espacial, o que sugere que há oscilações temporais dos investimentos municipais, corroborando o trabalho de Orair, Gouveia e Leal (2014), que confirma Investimentos públicos em períodos cíclicos cujas trajetórias coincidem com os períodos antes das eleições. Outro resultado interessante foi que as variáveis IFGF - Gastos com pessoal e IFGF - Liquidez apresentaram praticamente a mesma va- riação espacial e temporal. Isso poderia ser explicado por elas serem enfatizadas pelos municípios para o cumprimento da LRF.

Na literatura, segundo Brueckner (2003), a interação espacial entre municípios refere-se ao fato de que o incumbente de determinado município imita o padrão de gastos de seus vizinhos com o objetivo de sinalizar sua competência para seu eleitorado (Besley, 1995) ou atua como um difusor de investimentos, por exemplo. Nesse caso específico, o município que aumenta gastos em determinada área, por exemplo, o setor de saúde, pode fazer com que seus vizinhos reduzam os gastos nesse mesmo setor, pois o investimento naquela região não seria mais necessário (Case et al., 1993).

Os resultados da matriz de correlação evidenciaram a associação geral entre cada uma das variáveis da pesquisa, conforme apresentado na tabela 2.

Tabela 2. Matriz de correlação entre as variáveis da pesquisa

\begin{tabular}{|l|r|r|r|r|r|r|r|}
\hline & \multicolumn{1}{|c|}{ IFDM } & \multicolumn{1}{c|}{1} & \multicolumn{1}{c|}{2} & \multicolumn{1}{c|}{3} & \multicolumn{1}{c|}{4} & \multicolumn{1}{c|}{5} & 6 \\
\hline IFDM & \multicolumn{1}{|c|}{1} & & & & & & \\
\hline 1. Receita Própria & 0,5041 & 1 & & & & & \\
\hline 2. Gastos com pessoal & 0,1115 & 0,0701 & 1 & & & & \\
\hline 3. Investimentos & 0,1179 & 0,0743 & 0,2266 & 1 & & & \\
\hline 4. Liquidez & 0,2182 & 0,1717 & 0,1872 & 0,1384 & 1 & & \\
\hline 5. Custo da Dívida & $-0,0109$ & $-0,1044$ & 0,1115 & 0,1136 & 0,1023 & 1 & \\
\hline 6. PIB & 0,4939 & 0,6193 & $-0,0133$ & 0,0089 & 0,0963 & $-0,1443$ & 1 \\
\hline
\end{tabular}

Todas as Correlações foram significativas a 0,01.

Fonte: elaborado pelos autores 
As variáveis que apresentaram maior correlação significativa e positiva com o IFDM foram o IFGF - Receita Própria (0,5041); PIB Per capita Municipal $(0,4939)$ e IFGF Liquidez $(0,2181)$. As outras variáveis apresentaram baixa correlação significativa e positiva, destacando-se a correlação negativa da variável IFGF - Custo da dívida, quase próxima de zero $(-0,0109)$. Tal relação negativa sugere uma gestão ruim com relação aos empréstimos e financiamentos e que os municípios estão buscando financiamentos via empréstimos para subsidiar suas atividades. Observou-se também na variável IFGF - Custo da dívida correlação significativa e negativa com o IFGF - Receita própria $(-0,1044)$ e correlação significativa e negativa com o PIB Per capita Municipal $(-0,1443)$, o que reforça a sugestão do endividamento, pois aumentos no Custo da dívida tendem a repercutir em reduções na Receita própria e na renda per capita. Os resultados sugerem que a baixa capacidade de geração de receita própria média dos municípios brasileiros, evidenciada na análise descritiva, apresentou menor média e maior desvio padrão, denotando Gestão crítica (média de 0,2344 e variabilidade de $81,27 \%$ ). Isso poderia estar relacionado à busca por recursos de terceiros. Apesar disso, os resultados da análise descritiva sugerem que os municípios têm atendido a LRF no quesito Custo da dívida, pois tal indicador apresentou a maior média (média de 0,7996 e variabilidade de $21,73 \%$ ). Os resultados da análise de correlação seguiram a tendência esperada, pois corroboraram os estudos de Matias et al. (2002); Resende, Slomski, e Corrar (2005); Oliveira et al., (2010); Sousa et al., (2011); Oliveira e Silva (2012); Klering, Kruel, e Stranz (2012); Sousa et al., (2013); Duarte e Lima (2013); Denardi et al., (2014), Leite
Filho e Fialho (2014). Eles confirmaram haver associação entre as variáveis de gestão e de desenvolvimento dos municípios brasileiros.

Para verificar a relação entre indicadores de gestão pública municipal com base na LRF e nos indicadores de desenvolvimento municipal dos municípios brasileiros foi processada inicialmente a regressão linear múltipla com dados empilhados, em que se avaliaram os principais pressupostos teóricos de um modelo de regressão. Para testar a multicolinearidade da regressão de dados em painel, foi usada a estatística Variance Inflation Factor for the independente variables (VIF). Os resultados do teste, aliados à análise da matriz de correlação, sugeriram ausência de multicolinearidade. Outro teste analisou a normalidade dos resíduos. Optou-se pela realização do teste de normalidade procedendo a análise gráfica do ajuste dos resíduos e verificou-se uma distribuição aproximadamente normal, conforme densidade de estimativa de Kernel. Ao plotar o gráfico, obsevou-se que os resíduos seguiram uma distribuição aproximadamente normal. Verificaram-se ainda a Autocorrelação serial de dados em painel pelo teste de Wooldridge (Prob > F, 0,0000) e a heterocedasticidade de dados em painel pelo teste de Wald (Prob > chi2, 0,0000). Tais resultados sugeriram que há autocorrelação serial e que os dados são heterocedásticos.

Optou-se por processar as regressões de dados em painel de forma robusta, para correção dos efeitos da Autocorrelação serial e a heterocedasticidade de dados em painel. Foram processados três modelos de regressão de dados em painel, com as estimativas MQG, Efeitos Fixos e Efeitos Aleatórios, cujos resultados são descritos a seguir, na tabela 3: 
Tabela 3. Resultados das regressões de dados em painel, estimações por MQG, EF e EA

\begin{tabular}{|c|c|c|c|c|c|c|}
\hline \multirow[b]{2}{*}{ Variáveis / modelos } & \multicolumn{2}{|c|}{ MQG } & \multicolumn{2}{|l|}{ EA } & \multicolumn{2}{|l|}{ EF } \\
\hline & Coeficiente & P-Value & Coeficiente & P-Value & Coeficiente & P-Value \\
\hline Intercepto & 0,2437444 & 0,0000 & 0,355106 & 0,0000 & 0,5411044 & 0,0006 \\
\hline IFGF - Receita Própria & 0,1596903 & 0,0000 & 0,0419568 & 0,0000 & 0,0071784 & 0,0000 \\
\hline $\begin{array}{l}\text { IFGF - Gastos com } \\
\text { pessoal }\end{array}$ & 0,0308188 & 0,0000 & $-0,0268069$ & 0,0000 & $-0,0399736$ & 0,0000 \\
\hline IFGF - Investimentos & 0,0226707 & 0,0000 & 0,0077183 & 0,0000 & 0,0062767 & 0,0000 \\
\hline IFGF - Liquidez & 0,0327692 & 0,0000 & 0,0087124 & 0,0000 & 0,0047947 & 0,0000 \\
\hline IFGF - Custo da Dívida & 0,0237982 & 0,0000 & 0,0016208 & 0,4480 & $-0,0017177$ & 0,4200 \\
\hline PIB Per capita Mun. & 0,0236508 & 0,0000 & 0,0227828 & 0,0000 & 0,009047 & 0,0000 \\
\hline R2 Ajustado & \multicolumn{2}{|c|}{0,3374} & \multicolumn{2}{|c|}{0,3353} & \multicolumn{2}{|c|}{0,2268} \\
\hline Prob $>$ F ou Prob $>$ Chi2 & \multicolumn{2}{|c|}{0,0000} & \multicolumn{2}{|c|}{0,0000} & \multicolumn{2}{|c|}{0,0000} \\
\hline Observações & \multicolumn{2}{|c|}{31421} & \multicolumn{2}{|c|}{31421} & \multicolumn{2}{|c|}{31421} \\
\hline Grupos & \multicolumn{2}{|c|}{5555} & \multicolumn{2}{|c|}{5555} & \multicolumn{2}{|c|}{5555} \\
\hline
\end{tabular}

Fonte: elaborado pelos autores

Para se verificar qual das estimativas de regressão (MQG, EA e EF) apresentou os melhores resultados para estudar os efeitos dos indicadores de gestão pública municipal no desenvolvimento municipal, procedeu-se a testes de especificação dos modelos de regressão de dados em painel. Segundo Gujarati (2006, p. 524), "se o N (unidades de corte transversal) for pequeno e, neste caso, são cinco variáveis e o T grande (número de observações), neste caso foram 31421 observações, haverá diferenças significativas entre os modelos de regressão, e a escolha deverá ser feita por conveniência computacional.' Nesse caso, os dados se enquadram nas observações de Gujarati. Para se comparar o modelo de estimação por MQG com o EA, utilizou-se o teste do Multiplicador de Lagrange de Breusch e Pagan (LM). Os resultados: para um Chi2 $=0,07$ e Prob $>$ chi2 $=0,9658$, sugeriram que os estimadores por MQG foram mais eficientes que os estima- dores EA. Processou-se o teste de Chow para se comparar o modelo de estimação MQG com o EF. Para um F $(6,5555)=77,29$ e Prob $>F=0,0000$, aceita-se que os estimadores por MQG foram mais eficientes do que EF. Para se comparar o modelo de estimação por EA com EF, usou-se o teste de Hausman. Os resultados: para um Chi2 = 2316,57 e Prob > chi2 =0,0000, sugeriram que os estimadores por EF são mais eficientes que os estimadores EA. Assim, conforme os resultados dos testes computacionais sugeridos por Gujarati, o modelo de regressão escolhido foi o MQG.

Os resultados das estimações por MQG evidenciaram que o desenvolvimento municipal pode ser explicado em $33,75 \%$ pelo conjunto das variáveis de qualidade da gestão pública municipal em conjunto com o PIB Per capita. A estimação foi significativa a 0,01 (teste F) e foram observados coeficientes 
positivos e significativos a 0,01 para todas as variáveis do modelo de regressão, sendo os sinais dos parâmetros coerentes com as expectativas a priori, corroborando os resultados da matriz de correlação.

Os resultados da regressão de dados em painel evidenciaram relação positiva e significativa dos indicadores de gestão pública municipal com os indicadores de desenvolvimento dos municípios brasileiros, destacando-se principalmente a variável IFGF - Receita própria. Observou-se que todas as variáveis apresentaram coeficientes positivos e significativos, sugerindo que os municípios com melhores indicadores de gestão pública municipal, com base nos preceitos da LRF, teriam melhores indicadores de desenvolvimento econômico (IFGF - Receita própria, 0,1596903; IFGF -Liquidez, 0,0327692; IFGF - Gastos com pessoal, 0,0308188; PIB Per capita Mun., 0,0236508; IFGF - Custo da dívida, 0,0237982; IFGF Investimentos, 0,0226707). Tais pressuposições corroboraram os trabalhos de Matias et al. (2002); Resende, Slomski, e Corrar (2005); Oliveira et al., (2010); Sousa et al., (2011); Oliveira e Silva (2012); Klering, Kruel, e Stranz (2012); Sousa et al., (2013); Duarte e Lima (2013); Denardi et al., (2014), Leite Filho e Fialho (2014), nos quais os autores encontraram também evidências de que os aspectos da gestão pública, medidos por indicadores, influenciou o desenvolvimento econômico e social dos municípios.

Verificou-se que o IFGF - Receita própria foi a variável que apresentou maior coeficiente positivo e significativo na relação ao IFDM, denotando que tal indicador é a principal variável no modelo que explica o desenvolvimento municipal. Apesar de o baixo percen- tual de geração de receitas próprias (Pereira, 2012) ser uma característica da maioria dos municípios brasileiros, os resultados maior influência na variabilidade do indicador de desenvolvimento dos municípios.

As demais variáveis do IFGF demonstraram-se como coeficientes baixos, positivos e significativos na explicação do desenvolvimento dos municípios brasileiros. Tais resultados já eram esperados, uma vez que se partiu do pressuposto que, quanto mais favoráveis os indicadores de gestão pública municipal, meIhores seriam os indicadores de desenvolvimento. Reitera-se que o cumprimento dos pressupostos da LRF, buscando-se melhora nos indicadores de gestão pública, interfere positivamente no desenvolvimento dos municípios, explicando-o em 33,75\%.

A variável de controle (PIB Per capita) apresentou coeficiente positivo e significativo na explicação da relação entre a gestão pública municipal e o desenvolvimento. Tal direcionamento já era esperado, pois, na análise de correlação, esse indicador mostrou-se associado positivamente à variável de desenvolvimento. Tais resultados reforçam a ideia de que o PIB Per capita municipal exerceu efeitos positivos e significativos sobre a variável de desenvolvimento municipal, sugerindo, que aumentos nesse índice podem aumentar os indicadores de desenvolvimento municipal, confirmando os resultados dos estudos de Deininer e Squire, (1998); Ravallion e Chen, (1997); Easterly, (1999); Dollar e Kraay, (2002); Taques e Mazutti, (2010).

De uma forma geral, entende-se que os indicadores utilizados na pesquisa foram importantes para mensurar a qualidade da gestão fiscal municipal, com base nos preceitos da 
LRF, bem como para avaliar o desenvolvimento municipal em uma escala homogênea $(0$ a 1). Foram regulares em termos de tempo e séries históricas disponíveis (painel balanceado anual de 2006 a 2011) e compatíveis para a escala geográfica de abrangência (no âmbito municipal), permitindo comparações e estabelecimento de relações, atendendo os requisitos descritos por Januzzi (2001).

Destaca-se o caráter de originalidade da pesquisa. Apesar de haver outros estudos com diversos indicadores de gestão pública, sociais e de desenvolvimento, este estudo relacionou o IFGF com o IFDM numa perspectiva municipal de abrangência nacional e num período contínuo de seis anos, contribuindo para discussão do uso dos indicadores de gestão, indicadores sociais e de desenvolvimento.

\section{Conclusão}

O objetivo principal desta pesquisa foi verificar a relação entre indicadores de gestão pública municipal, com base na LRF e nos indicadores de desenvolvimento municipal dos municípios brasileiros.

Por meio da análise descritiva dos dados, a perspectiva temporal dos municípios brasileiros de 2006 a 2011 apresentou-se com desenvolvimento médio moderado, e os indicadores de gestão pública municipal foram considerados de gestão em dificuldade crítica, excluindo-se o custo da dívida e os gastos com pessoal, variáveis que são mais observadas pela LRF pelos municípios ao longo do tempo. Observou-se que os municípios apresentam oscilações temporais nos investimentos públicos municipais, em perí- odos cíclicos, cujas trajetórias coincidem com os períodos antes das eleições. Verificou-se ainda que houve interação espacial entre os municípios, ou seja, os municípios vizinhos se influenciam.

As variáveis da pesquisa apresentaram correlação significativa e positiva com o IFDM, destacando-se o IFGF - Receita própria $(0,5041)$; PIB Per capita Municipal $(0,4939)$ e IFGF Liquidez $(0,2181)$. As outras variáveis apresentaram baixa correlação significativa e positiva, destacando-se a correlação negativa da variável IFGF - Custo da dívida, quase próxima a zero $(-0,0109)$. Tal relação negativa sugere uma gestão ruim com relação aos empréstimos e financiamentos e que os municípios estão buscando financiamentos via empréstimos para subsidiar suas atividades. Observou-se também na variável IFGF - Custo da dívida correlação significativa e negativa com o IFGF - Receita própria $(-0,1044)$ e correlação significativa e negativa com o PIB Per capita Municipal $(-0,1443)$, o que reforça a sugestão do endividamento, pois aumentos no Custo da dívida tendem a repercutir em reduções na Receita própria e na renda per capita.

Os resultados das regressões de dados em painel evidenciaram que o desenvolvimento municipal pode ser explicado em $33,75 \%$ pelo conjunto das variáveis de gestão pública municipal em conjunto com o PIB Per capita municipal. A estimação foi significativa e foram observados coeficientes positivos e significativos para todas as variáveis do modelo de regressão, sendo os sinais dos parâmetros coerentes com as expectativas a priori, corroborando os resultados da matriz de correlação (IFGF - Receita própria, 0,1596903; IFGF - Liquidez, 0,0327692; 
IFGF -Gastos pessoal, 0,0308188; PIB Per capita Mun., 0,0236508; IFGF - Custo da Dívida, 0,0237982; IFGF - Investimentos, 0,0226707 ). Conclui-se assim que houve relação positiva e significativa entre os indicadores de gestão pública municipal, com base na LRF e nos indicadores de desenvolvimento municipal. Tais pressuposições corroboraram os estudos anteriores citados, nos quais os autores encontraram também evidências de que aspectos da gestão pública, medida por indicadores, influenciou o desenvolvimento econômico e social dos municípios.

\section{Referências}

Brasil (2000). Lei Complementar n 101, de 4 de Maio de 2000. Diário Oficial da União, Brasília, DF, 5 maio 2000. Recuperado de http://pesquisa.in.gov.br/imprensa/jsp/visualiza/index.jsp?data $=05 / 05 / 2000 \&$ jornal $=1 \& p$ agina $=82 \&$ totalArquivos $=152$

Besley, T. (1995). Property rights and investment incentives: Theory and evidence from Ghana. Journal of Political Economy, 103, 903-937

Brueckner, J. K. (2003). Strategic interaction among governments: An overview of empirical studies. International Regional Science Review, 26 (2), 175-188.

CNM - Conselho Nacional dos Municípios (2014). Recuperado de www.cnm.org.br

Case, A., Rosen, H. S., \& Hines, J. R. (1993). Budget spillovers and fiscal policy interdependence: Evidence from the states. Journal of Public Economics, 52, 285-307.
Costa, I. S. (2011). Gasto público e desenvolvimento socioeconômico: Uma análise dos municípios de estado do Paraná. Dissertação de mestrado. Universidade Federal de Viçosa, Viçosa, Brasil.

Denardi, R. A., Henderikx, E. M. G. J., CastiIhos, D. S. B de., \& Bianchini, V. (2014). Fatores que afetam o desenvolvimento local em pequenos municípios do estado do Paraná. Recuperado de www.gpublic.info/sites/default/files/biblioteca/desen_fatores.pdf

Dollar, D., \& Kraay, A. (2002). Growth is good for the poor. Journal of Economic Growth, 7(3), 195-225.

Duarte, A., \& Lima, D. (2013, 21 de setembro). Boa gestão fiscal melhora indicadores sociais dos municípios. Recuperado de www. oglobo.globo.com/pais/boa-gestao-fiscal-melhora-indicadores-sociais-de-municipios-10083540 ? service $=$ print

Ervilha, G. T., Alves, F. F., \& Gomes, A. P. (2013). Desenvolvimento municipal e eficiência dos gastos públicos na Bahia: Uma análise do IFDM a partir da metodologia DEA. Revista Bahia Análise \& Dados, 23(3), 553-566.

Easterly, W. (1999). Life during growth: International evidence on quality of life and per capita income. Journal of Economic Growth, 4(1), 1-19.

Firjan - Federação das Indústrias do Estado do Rio de Janeiro. (2014). Recuperado de www.Firjan.org.br/data/pages/2C908CE923 4D9BDA01234E532B007D5D.htm

Firovante, D. G., Pinheiro, M. M. S., \& Vieira, $\mathrm{R}$ da S. (2006). Lei de responsabilidade 
fiscal e finanças públicas municipais: Impactos sobre despesa com pessoal e endividamento. IPEA. Recuperado de http:// agencia.ipea.gov.br/index.php?option=com content\&view=article\&id=4803:td-1223-lei-de-responsabilidade-fiscal-e-financas-publicas-municipais-impactos-sobre-despesas-com-pessoal-e-endividamento\&catid= 308:2006\&directory $=1$

Guimarães, S. C. (2008). Avaliação de desempenho da gestão pública municipal. Dissertação de Mestrado. Fundação Getúlio Vargas - FGV. (Mestrado em Administração Pública). São Paulo.

Guimarães, J. R. S.; Jannuzzi, P. de M. (2004). Indicadores sintéticos no processo de formulação e avaliação de políticas públicas: Limites e legitimidades. In: XIV Encontro Nacional de Estudos Populacionais, ABEP. Caxambu (MG).

Hair Jr., J. F., Black, R. E., \& Anderson, R. L. T. (2005). Análise multivariada de dados. 5. ed. Porto Alegre: Bookman, 593 p.

IETS - Instituto de Estudos do Trabalho e Sociedade (2013). Recuperado de www. iets.org.br/projeto/desenvolvimento-com-justica-social-uma-agenda-para-os-municipios

Jannuzzi, P. de M. (2001). Indicadores Sociais no Brasil. Campinas: Ed. Alínea.

Jannuzzi, P. de M. (2002). Considerações sobre o uso, mau uso e abuso dos indicadores sociais na formulação e avaliação de políticas públicas municipais. Revista Brasileira de Administração Pública, Rio de Janeiro, 36(1):51-72, jan/fev.
Kayano, J.; Caldas, E. de L. (2002). Indicadores para o diálogo. GT Indicadores. Plataforma Contrapartes Novib. Série Indicadores, 08. São Paulo.

Klering, L. R., Kruel, A. J., \& Stranz, E. (2012). Os pequenos municípios do Brasil - uma análise a partir de índices de gestão. Análise - Revista de Administração da PUCRS, 23(1), 31-44.

Klering, L. R., Stranz, E., \& Gobetti, S. W. (2007). Avaliação da Gestão dos Municípios do Brasil pelo IRFS - Índice de Responsabilidade Fiscal, de Gestão e Social - 2002 a 2006. Revista Redes,12(2), 196-217.

Leite Filho, G. A., \& Fialho, T. M. M. (2014) Associação entre Indicadores de Gestão Pública municipal e Indicadores de Desenvolvimento dos Municípios Brasileiros. Anais do Congresso USP de Controladoria e Contabilidade, São Paulo, Brasil, 14.

Matias, A. B., Ortolani, F., \& Campello, C. A. G. B. (2002). A relação (in)existente entre a responsabilidade fiscal e responsabilidade social nos municípios paulistas. Anais do Assembly of the Latin American Council of College of Business Administration - CLA$D E A$, Porto Alegre, Brasil, 37.

Matias-Pereira, J. (2012). Manual de Gestão Pública Contemporânea. 4 ed. São Paulo: Atlas.

Meirelles, H. L. (2002). Direito administrativo brasileiro. 5 ed. São Paulo: Saraiva.

Mendes, M. (2011, 28 junho). Porque é importante controlar o gasto público? Recupe- 
rado de www.brasil-economia-governo.org. br/2011/06/28/por-que-e-importante-controlar-o-gasto-publico

Mendes, J. M. G.; Rezende, D. A. (2008). A influência dos indicadores sócio-econômicos na gestão municipal para o desenvolvimento local. Anais. XVI Encontro Nacional de Estudos Populacionais, ABEP, realizado em Caxambú (MG) - Brasil.

Nahas, M. I. P.; Gonçalves, E.; Souza, R. G. V. de; Vieira, C. M. (2006). Sistemas de indicadores Municipais no Brasil: Experiências e metodologias. Anais do XV Encontro Nacional de Estudos Populacionais, $A B E P$, realizado em Caxambu (MG) - Brasil, 18 a 22 de setembro.

Oliveira, M. de J., \& Silva E. A. (2012). Eficiência na Gestão Fiscal Pública e o Desenvolvimento Socioeconômico dos Municípios da Microrregião de Cataguases/MG. Anais do Encontro de Administração Pública e Governo - EnApg, Salvador, Brasil, 5.

Oliveira, F. H., Peter, M. G. A., \& Meneses, A. F. (2009). Lei de responsabilidade fiscal: Implicações nos indicadores sociais municipais. Anais do Congresso USP de Controladoria e Contabilidade, São Paulo, Brasil, 9.

Orair, R. O., Gouvêa, R. R., \& Leal, E. M. (1999). Ciclos políticos eleitorais e investimentos das administrações públicas. Brasília: IPEA.

Rezende, A. J., Slomski, V., \& Corrar, L. J. (2005). A gestão pública municipal e a eficiência dos gastos públicos: Uma investigação empírica entre as políticas públicas e o índice de desenvolvimento humano (IDH) dos municípios do estado de São Paulo. Revista Universo Contábil, 1(1), 24-40.

Ravallion, M.; Chen, S. (1997). What can new survey data tell us about recent changes in distribution and poverty? World Bank Economic Review, 11(2), 357-382.

Scarpin, J. E.; Slomski, V. (2007). Estudo dos fatores condicionantes do índice de desenvolvimento humano nos municípios do estado do Paraná: Instrumento de controladoria para a tomada de decisões na gestão governamental. Revista de Administração Pública, 41(5), 909-933.

Siedenberg, D. R. (2003). Indicadores de desenvolvimento socioeconômico: Uma síntese. Revisa Desenvolvimento em Questão, 1(1), 45-71.

Silva, B. 1995. Teoria das Funções Municipais. 2 ed. Rio de Janeiro: IBAM.

Sousa, P. F. B., Gomes, A. de O., Lima, A. O., Peter, M. da G. A., \& Machado, M. V. V. (2011). Lei de responsabilidade fiscal e sua relação com o desenvolvimento municipal: Uma análise utilizando dados em painel. Anais do Congresso Brasileiro de Custos - CBC, Rio de Janeiro, Brasil, 18.

Sousa, P. F. B de, Lima, A. O., Nascimento, C. P. S. do. Peter, M. G. A., Machado, V. V. M., \& Gomes, A de O. (2013). Desenvolvimento municipal e cumprimento da lei de responsabilidade fiscal: Uma análise dos municípios brasileiros utilizando dados em painel. Revista evidenciação contábil \& finanças, $1(1), 58-70$.

Taques, F. H., \& Mazutti, C. C. de T. P. da C. 
(2010). Qual a relação entre desigualdade de renda e nível de renda per capta? Testando a hipótese de Kuznets para as unidades federativas brasileiras. Planejamento e Políticas Públicas, 35(1), 20-36.

Viana, A., Silva, E. S., Almeida, F. P. A., Anjos Junior, O. R., Silva, L. A. S. C., Rodrigues, M. P., Costa Neta, R. L., Figueiredo, N. R M., \&
Santana, F. L. (2011). Eficiência dos gastos públicos de João Pessoa: Um estudo empírico entre as políticas públicas de educação e saúde dos municípios da região metropolitana. Recuperado de www.recantodasletras. com.br/arti-gos/3087722 